\title{
Effect of interfacial intermixing on the Dzyaloshinskii-Moriya interaction in $\mathrm{Pt} / \mathrm{Co} / \mathrm{Pt}$
}

\author{
Adam W. J. Wells, ${ }^{*}$ Philippa M. Shepley, Christopher H. Marrows, and Thomas A. Moore \\ School of Physics and Astronomy, University of Leeds, Leeds LS2 9JT, United Kingdom \\ (Received 12 August 2016; revised manuscript received 20 January 2017; published 21 February 2017)
}

\begin{abstract}
We study the effect of sputter-deposition conditions, namely, substrate temperature and chamber base pressure, upon the interface quality of epitaxial $\mathrm{Pt} / \mathrm{Co} / \mathrm{Pt}$ thin films with perpendicular magnetic anisotropy. Here we define interface quality to be the inverse of the sum in quadrature of roughness and intermixing. We find that samples with the top $\mathrm{Co} / \mathrm{Pt}$ layers grown at $250^{\circ} \mathrm{C}$ exhibit a local maximum in roughness intermixing and that the interface quality is better for lower or higher deposition temperatures, up to $400{ }^{\circ} \mathrm{C}$, above which the interface quality degrades. Imaging the expansion of magnetic domains in an in-plane field using wide-field Kerr microscopy, we determine the interfacial Dzyaloshinskii-Moriya interaction (DMI) in films in the deposition temperature range $100^{\circ} \mathrm{C}$ to $300^{\circ} \mathrm{C}$. We find that the net DMI increases as the difference between top and bottom Co interface quality increases. Furthermore, for sufficiently low base pressures, the net DMI increases linearly with the deposition temperature, indicating that fine-tuning of the DMI may be achieved via the deposition conditions.
\end{abstract}

DOI: 10.1103/PhysRevB.95.054428

\section{INTRODUCTION}

The interfacial Dzyaloshinskii-Moriya interaction (DMI) is a key ingredient in determining the equilibrium domain wall (DW) spin structure in thin magnetic films with perpendicular anisotropy and structural inversion asymmetry, such as $\mathrm{Pt} / \mathrm{Co} / \mathrm{AlO}_{\mathrm{x}}$ [1-3], $\mathrm{Pt} / \mathrm{Co} / \mathrm{Ir}[4,5], \mathrm{Pt} /[\mathrm{Co} / \mathrm{Ni}]$ [6-8], etc. The DW spin structure in turn determines how the DW responds to a driving force. In the presence of DMI, bubble domains expand asymmetrically in simultaneously applied in-plane and out-of-plane fields [4,9-12], which enables evaluation of the DMI and of the DW spin structure. For sufficiently large DMI, Néel walls are stable $[13,14]$ and have been found to move at several hundred meters per second under spin-orbit torque $[7,11,14]$. Beyond that, skyrmions may be stabilized [15-19] and could have a huge impact on magnetic memory $[20,21]$ and logic devices [22,23].

Since DMI originates at the interfaces of a thin magnetic film [24], contributions from the top and bottom interfaces must differ in magnitude or sign to effect a net DMI. Previously, it has been shown that even nominally symmetric $\mathrm{Pt} / \mathrm{Co} / \mathrm{Pt}$ possesses DMI $[4,9,10,25,26]$. Bubble domains in room-temperature sputtered $\mathrm{Pt} / \mathrm{Co} / \mathrm{Pt}$ on a silicon substrate expand asymmetrically in an applied in-plane field [4], indicating a net DMI. However, if $\mathrm{Pt} / \mathrm{Co} / \mathrm{Pt}$ is grown epitaxially on sapphire, the domain expansion can be symmetric [4], indicating that there is no net DMI in this case. This highlights the importance of structure and the relative interface morphology of upper and lower Co interfaces in determining the DMI.

Here we adjust the interface morphology of the upper Co interface relative to the lower Co interface by controlling the substrate temperature during deposition. We find that Co-Pt intermixing increases in the temperature range $100{ }^{\circ} \mathrm{C}-250^{\circ} \mathrm{C}$ and correlates with an increased magnitude of the DMI field, which is more pronounced at lower base pressures. Our results show that the interfacial DMI depends very sensitively on the ferromagnet/heavy-metal interface morphology and thus on film deposition conditions such as substrate temperature and chamber pressure.

*pyaww@leeds.ac.uk

\section{SAMPLE DEPOSITION}

The setup of the deposition chamber is as follows (see also Supplemental Material [27]): the substrates are held on a sample wheel, and each is heated by bulbs from behind. The sample wheel rotates above a ring of sputtering guns with a shutter wheel in between. Permanent magnets atop the shutter wheel generate a radial magnetic field of $14.5 \mathrm{mT}$ above the aperture through which sputtering takes place. Atop each gun is an individual shutter which determines the deposition time.

The samples investigated here are composed of a $\mathrm{Pt}(3$ $\mathrm{nm}) / \mathrm{Co}(0.7 \mathrm{~nm}) / \operatorname{Pt}(1 \mathrm{~nm})$ epitaxial stack deposited by dcmagnetron sputtering onto a $C$-plane sapphire $\left(\mathrm{Al}_{2} \mathrm{O}_{3}\right)(0001)$ substrate previously annealed at $700{ }^{\circ} \mathrm{C}$ for $4 \mathrm{~h}$. The $\mathrm{Pt}$ seed layer was sputtered with the substrate held at $550^{\circ} \mathrm{C}$ for optimum smoothness [28]. Measurements of the residual resistivity ratio (RRR) and the full width at half maximum (FWHM) of the $\mathrm{x}$-ray diffraction rocking curves in previous work [28] showed that sputtering onto a $C$-plane sapphire substrate held at a temperature between $450^{\circ} \mathrm{C}$ and $550{ }^{\circ} \mathrm{C}$ yielded high-quality crystalline $\mathrm{Pt}$ films. The Co and top Pt layer were then sputtered with the substrate held at a temperature in the range $50{ }^{\circ} \mathrm{C}-500{ }^{\circ} \mathrm{C}$ to aid epitaxy, focusing between $100^{\circ} \mathrm{C}$ and $300^{\circ} \mathrm{C}$, where a high degree of crystallographic ordering was found previously [28]. Films were grown with the base pressure in the range $1.1 \times 10^{-7}$ to $3.3 \times 10^{-7}$ Torr, measured immediately prior to deposition of the seed layer. The Ar pressure needed to obtain stable plasmas was $2.7 \times 10^{-3}$ to $3.1 \times 10^{-3}$ Torr.

In a typical deposition run, a set of samples was grown with the Co and top Pt layer of the first sample deposited at the highest temperature (e.g., $300^{\circ} \mathrm{C}$ ) and of the last sample deposited at the lowest temperature (e.g., $100^{\circ} \mathrm{C}$ ). This typical set, which we term type A, forms the basis of this study: detailed structural characterization of these films is reported in Sec. III, which permits conclusions to be drawn about the dependence of the DMI on the interface morphology, reported in Sec. V. Other sets of samples were grown in order to determine the contribution of each interface to the perpendicular anisotropy, reported in Sec. IV. To keep the bottom Pt/Co interface morphology constant and modify only the top $\mathrm{Co} / \mathrm{Pt}$ interface, a set was grown with the Co layer 


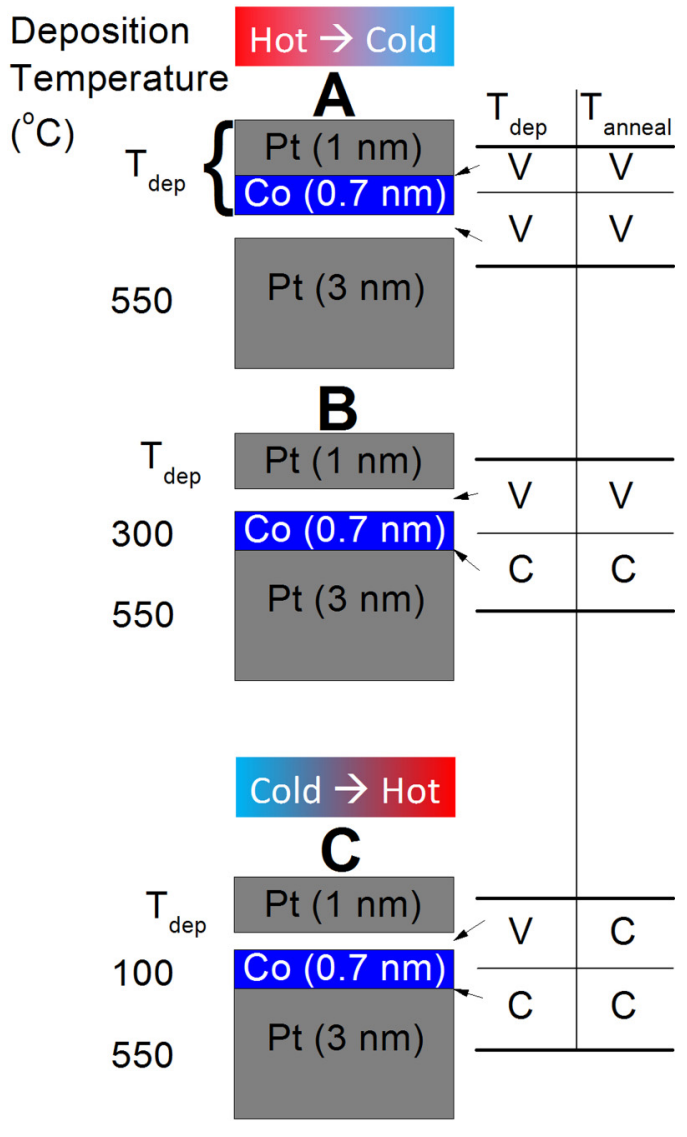

FIG. 1. Definition of sample sets A, B, and C according to which deposition conditions vary $(\mathrm{V})$ or stay constant $(\mathrm{C})$ between samples, with respect to the top and bottom Co interfaces in a $\operatorname{Pt}(3$ $\mathrm{nm}) / \mathrm{Co}(0.7 \mathrm{~nm}) / \mathrm{Pt}(1 \mathrm{~nm})$ stack. The deposition conditions indicated are the deposition temperature $T_{\text {dep }}$ and the annealing temperature $T_{\text {anneal }} . T_{\text {anneal }}$ is defined as the highest temperature experienced by the completed stack.

deposited at a fixed temperature and the deposition temperature of the top Pt layer varied (type B). A third set was grown akin to type B but with the order of deposition reversed such that the lowest-temperature samples were grown first and the highest-temperature samples were grown last (type C). It was deduced that the anisotropy originates at both top and bottom Co interfaces and that it is unaffected by differences in interface quality in the type-A films, allowing conclusions about the DMI to be drawn for a set of films where the interface quality varies but the anisotropy remains constant.

All samples in the growth chamber are heated simultaneously, so the maximum temperature a sample experiences may be different from its deposition temperature $T_{\mathrm{dep}}$, and this we term the annealing temperature $T_{\text {anneal }}$. For example, in films of type $\mathrm{C}$, samples grown at $100^{\circ} \mathrm{C}$ at the beginning of the run have effectively been annealed at $300^{\circ} \mathrm{C}$ by the end of the run, whereas for films of types $\mathrm{A}$ and $\mathrm{B}, T_{\text {anneal }}$ is always equal to $T_{\mathrm{dep}}$. Figure 1 organizes information by interface on the deposition temperature and annealing temperature of each set of films. We also consider the chamber base pressure $P_{\text {base }}$ to be a deposition condition: while the recorded base pressure is that obtained immediately prior to deposition of the Pt seed layer, $P_{\text {base }}$ in fact decreases gradually during a deposition run as a result of gettering and diminishing outgassing, and for the formation of a given interface it either varies or is constant across a sample set, following the pattern of $T_{\mathrm{dep}}$ indicated in Fig. 1. In Sec. V we show that $P_{\text {base }}$ determines how strongly the DMI is influenced by $T_{\text {dep }}$.

\section{STRUCTURAL CHARACTERIZATION}

Figure 2 shows high-angle x-ray diffraction (XRD) $\theta-2 \theta$ scans for type-A samples with the Co and top Pt layer deposited at $100{ }^{\circ} \mathrm{C}$ and $250{ }^{\circ} \mathrm{C}$ at the low and high ends of the $T_{\text {dep }}$ range that we focus on, measured using $\mathrm{Cu} K_{\alpha 1}$ $\mathrm{X}$ rays. There are peaks corresponding to fcc Pt (111) that obscure any Co (111) or (0001) peaks; the lack of any further peaks associated with Co or Pt confirms the epitaxial nature of the samples at low and high $T_{\text {dep }}$. The peaks for the sample deposition at the higher temperature $\left(250^{\circ} \mathrm{C}\right)$ display better-defined Kato fringes, indicating a more homogeneous layer thickness between the interfaces of the $\mathrm{Pt}$, consistent with higher atomic mobility during deposition. Since Pt is present at all interfaces in the stack, no information about individual interfaces is gained at this stage.

To investigate the dependence of the interface roughness on $T_{\text {dep }}$, rocking curves [Fig. 3(a)] were measured at the first Kiessig fringe of an X-ray reflectivity scan [e.g., at the $2.7^{\circ}$ position of the graph in Fig. 4(a)]. The peak and shoulders of the rocking curve include contributions from all layers, although the surface and bottommost interfaces are dominant [29]. This allows the Born approximation to be employed, where multiple reflections are disregarded. Here we define roughness as the collective deviation of atoms from an atomically sharp interface and intermixing as the deviation of individual atoms. The ratio of diffuse to specular area can be used to assess the roughness since it is the interfacial roughness which effectively transfers intensity from the specular peak to the diffuse component [30].

An average of the background intensity was taken, and the FWHM of the diffuse scattering was measured halfway between this value and the value for the horizontal flat of the

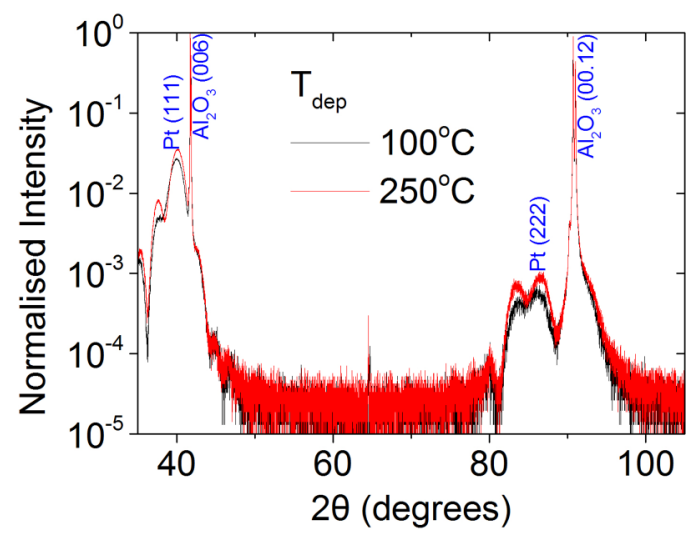

FIG. 2. High-angle XRD scans showing the epitaxial nature of the $\mathrm{Pt} / \mathrm{Co} / \mathrm{Pt}$ film for low and high deposition temperatures. The $\mathrm{Pt}$ interfaces are of sufficient quality to produce Kato fringes due to Pendellösung effects. The Co peaks are obscured by the Pt peaks. The low-intensity peak at $65^{\circ}$ is thought to be a higher-order substrate peak. 
(a)

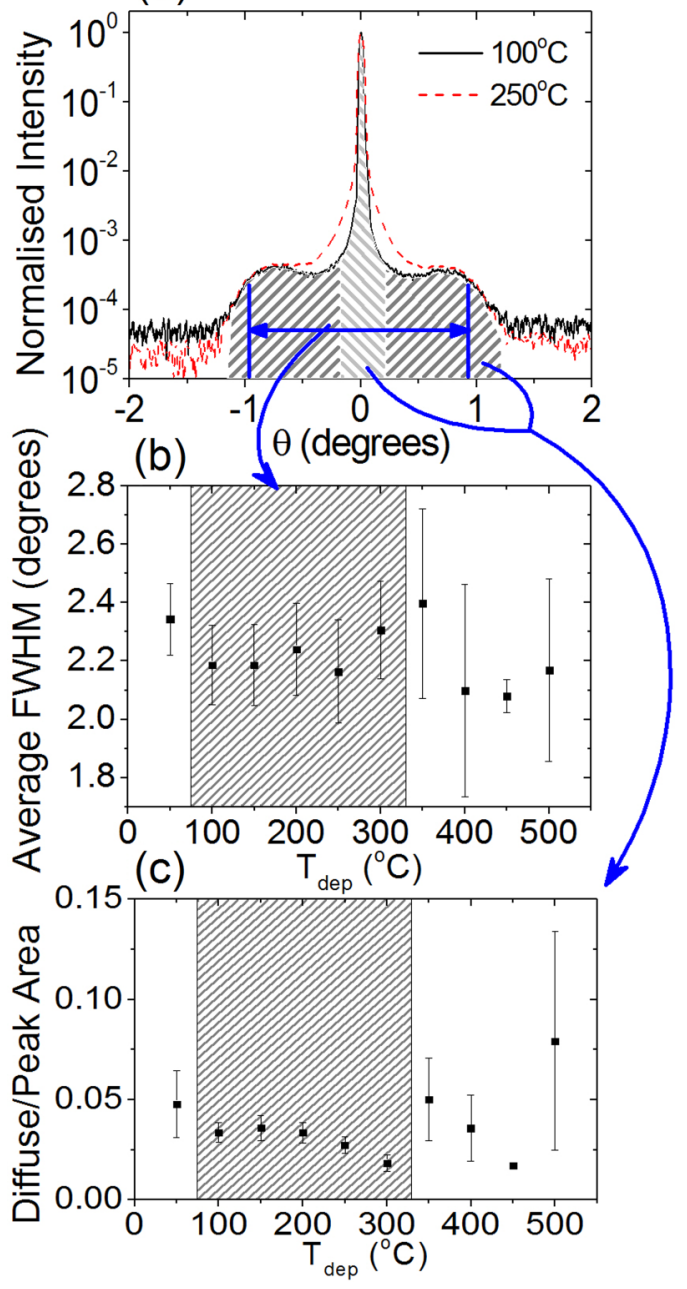

FIG. 3. (a) Analysis of roughness in $\mathrm{Pt} / \mathrm{Co} / \mathrm{Pt}$ deposited at different temperatures from rocking curves on the first Kiessig fringe. Representative data for low and high $T_{\text {dep }}$ are shown. The light and dark shaded areas represent the areas under the peak and diffuse wings, respectively. (b) FWHM of the diffuse background. (c) Ratio of the area under the diffuse section to that under the peak. The shaded areas in (b) and (c) highlight the main region of interest where the film is epitaxial with distinct layers.

shoulders. Figure 3(b) shows no change within the error of the FWHM of the diffuse scattering, which, when combined with the shape, indicates that the diffuse background is dominated by Yoneda scattering and is therefore defined by the refractive indices of the materials in the multilayer. In Fig. 3(c) the slight decrease of the diffuse/specular area in the primary temperature range of $100{ }^{\circ} \mathrm{C}$ to $300{ }^{\circ} \mathrm{C}$, however, indicates a decrease in the amount of roughness as the deposition temperature increases, probably due to the increasing mobility of the atoms, permitting a lower density of areas of roughness.

$\mathrm{X}$-ray reflectivity scans $(\theta-2 \theta)$ at low angles were fitted using BEDE REFS [31], as shown in Fig. 4(a). The sum in quadrature of roughness and intermixing $\sigma$ was used as an indicator of interface quality (small $\sigma$ indicating a high-quality interface) since the wave transfer vector remains normal to the surface in $\theta-2 \theta$ scans, making roughness and intermixing
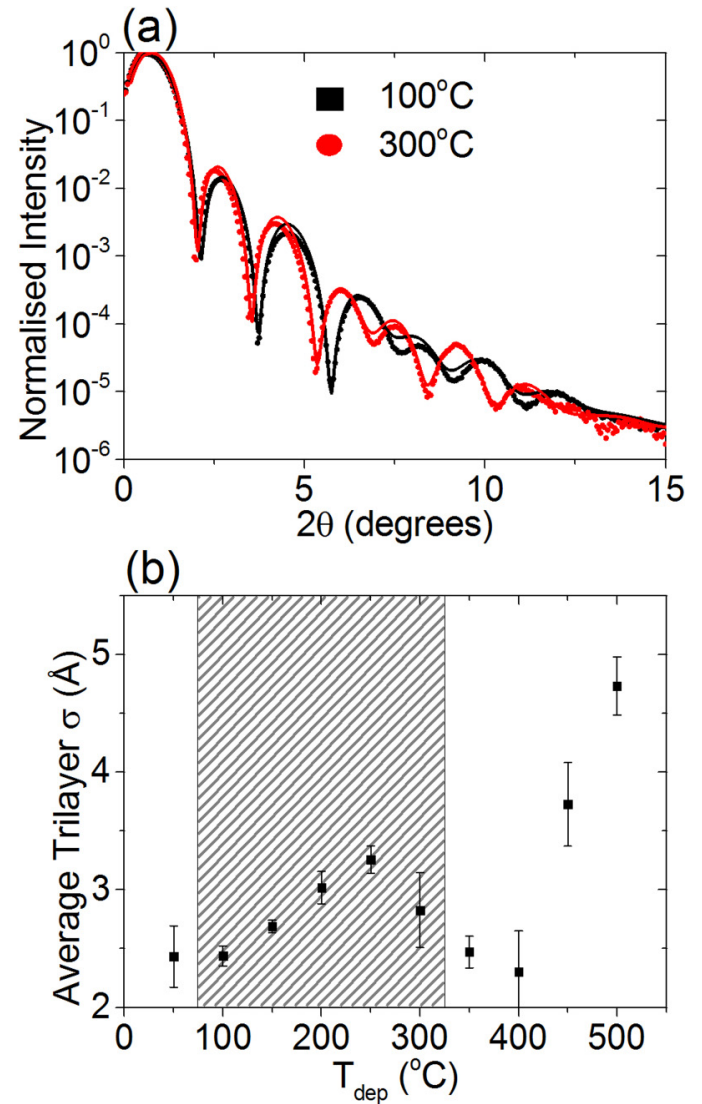

FIG. 4. (a) Low-angle $\mathrm{x}$-ray scans for $\mathrm{Pt} / \mathrm{Co} / \mathrm{Pt}$ deposited at low and high temperatures. The lines are from specular fits to the data using BEDE REFS. (b) $\sigma$ as a function of $T_{\text {dep }}$, averaged over all samples deposited at each temperature. The error bars are the larger of either statistical standard error from all samples at that temperature or propagated model fitting uncertainty. The shaded area in (b) indicates the main region of interest where the film is epitaxial with distinct layers.

indistinguishable. In Fig. 4(b) $\sigma$ averaged over the $\mathrm{Pt} / \mathrm{Co} / \mathrm{Pt}$ structure is plotted as a function of $T_{\text {dep }}$ for sample sets of type A. The general increase in $\sigma$ with temperature seen in Fig. 4(b) for deposition temperatures $50{ }^{\circ} \mathrm{C}-250{ }^{\circ} \mathrm{C}$ is opposite to the trend in roughness seen in Fig. 3(c), so we may deduce that it is predominantly due to an increase in Co-Pt intermixing. For $T_{\text {dep }}$ from $50{ }^{\circ} \mathrm{C}$ to $250{ }^{\circ} \mathrm{C}$, therefore, the $\mathrm{Pt} / \mathrm{Co} / \mathrm{Pt}$ becomes increasingly intermixed until at $250^{\circ} \mathrm{C}$ it reaches the point where the decrease of roughness outweighs any increase in intermixing, as indicated by a decrease in $\sigma$ above $250^{\circ} \mathrm{C}$.

According to equilibrium phase diagrams [32], our $T_{\text {dep }}$ is never quite high enough to produce $L 1_{0}$ ordered alloys, but above $400{ }^{\circ} \mathrm{C}$, they indicate that a disordered fcc CoPt alloy is grown rather than distinct, ordered fcc Co and Pt layers, and we suppose that this is what causes the sharp upturn in $\sigma$ for deposition temperatures $\geqslant 400^{\circ} \mathrm{C}$.

The structural characterization thus shows that it is possible to have some control over the overall interface quality in epitaxial $\mathrm{Pt} / \mathrm{Co} / \mathrm{Pt}$ via $T_{\text {dep }}$. While the average $\sigma$ value increases, the roughness decreases slightly as the deposition temperature of the Co and top Pt layer increases from $50{ }^{\circ} \mathrm{C}$ to $300^{\circ} \mathrm{C}$, from which we deduce that Co-Pt intermixing 
(a)

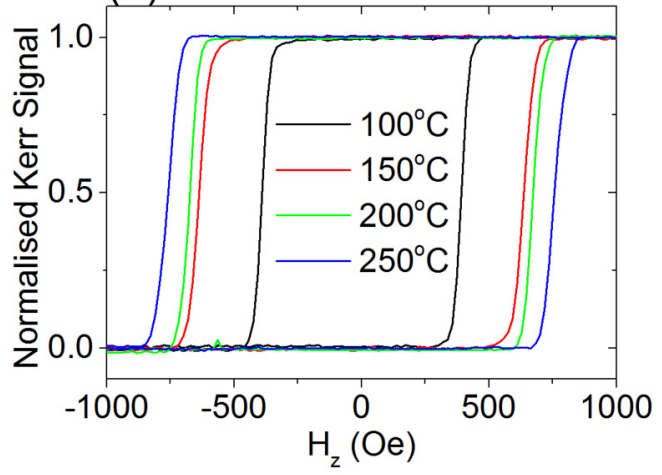

(b)

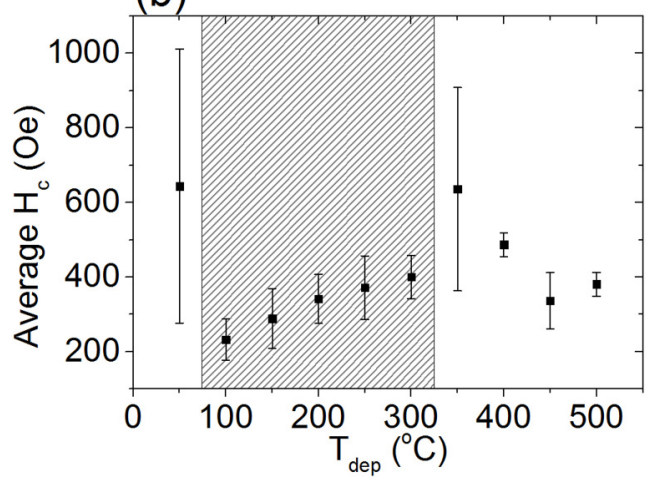

FIG. 5. (a) Polar MOKE hysteresis loops for $\mathrm{Pt} / \mathrm{Co} / \mathrm{Pt}$ deposited at different temperatures. (b) Coercive field as a function of $T_{\text {dep }}$, averaged over all samples deposited at each temperature. The shaded area indicates the main region of interest where the film is epitaxial with distinct layers.

contributes most to the increase in $\sigma$. We show in Secs. IV and $\mathrm{V}$, respectively, that this change in interface quality does not significantly affect the perpendicular anisotropy but that it is linked to changes in the net Dzyaloshinskii-Moriya interaction, indicating that, as $T_{\text {dep }}$ is increased, the qualities of the top and bottom Co interfaces do not change at the same rate.

\section{MAGNETIC CHARACTERIZATION}

To investigate the magnetic properties of the type-A films, hysteresis loops were measured using the polar magnetooptical Kerr effect (MOKE) with field applied perpendicular to the plane of the film. As shown in Fig. 5(a), for samples deposited at temperatures in the range $100{ }^{\circ} \mathrm{C}-250{ }^{\circ} \mathrm{C}$, the loops are square, indicating that perpendicular anisotropy dominates. Vibrating sample magnetometry measurements show that $M_{\mathrm{s}}$ is the same for all uncertainties of measurement, $95 \pm 6 \mu \mathrm{emu}$. The coercivity, shown in Fig. 5(b), increases as a function of $T_{\text {dep }}$ in a similar range of deposition temperatures as the interface quality $(1 / \sigma)$ decreases, as measured in the previous section. We speculate that the intermixing of Co and $\mathrm{Pt}$ that increases in the $T_{\mathrm{dep}}$ range $100{ }^{\circ} \mathrm{C}-300{ }^{\circ} \mathrm{C}$ leads to an effective broadening and smoothing of the magnetic (rather than chemical) interface that reduces the density and strength of magnetic defects, which in turn leads to larger nucleation fields for reverse domains and hence larger coercivities. This

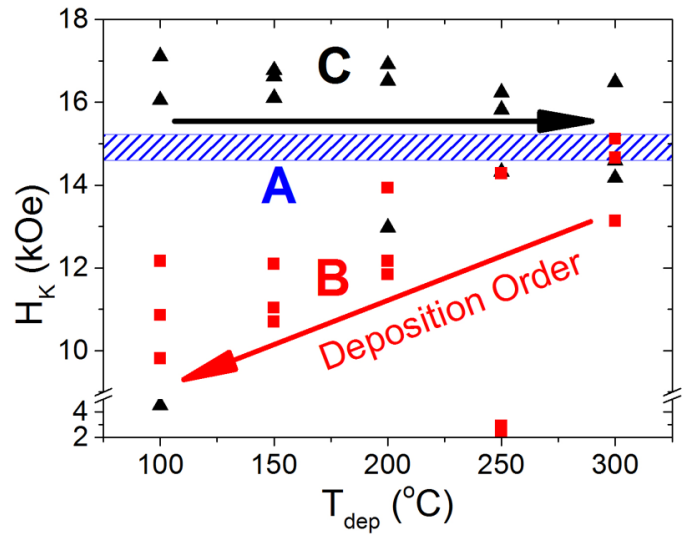

FIG. 6. Effective anisotropy field $H_{\mathrm{K}}$ for $\mathrm{Pt} / \mathrm{Co} / \mathrm{Pt}$ where the deposition temperature of the top $\mathrm{Co} / \mathrm{Pt}$ layers was varied (type $\mathrm{A}$ ) and where only the deposition temperature of the top Pt layer was varied (types $\mathrm{B}$ and $\mathrm{C}$ ). The hatched area indicates $H_{\mathrm{K}}$ measured for type-A films, including uncertainty. The arrows show the order of deposition: $300^{\circ} \mathrm{C}-100{ }^{\circ} \mathrm{C}$ (type $\mathrm{B}$, squares) and $100{ }^{\circ} \mathrm{C}-300^{\circ} \mathrm{C}$ (type $\mathrm{C}$, triangles). Error bars are smaller than or comparable to symbol sizes.

agrees with our findings of a more homogeneous Co layer thickness and a lower density of areas of roughness at the upper end of the $T_{\text {dep }}$ range.

To determine the perpendicular anisotropy, the sample was rotated through an angle $\theta$ about an in-plane axis in a constant magnetic field while the voltage $V$ associated with the extraordinary Hall effect was measured. The resulting $V-\theta$ data enabled calculation of the effective anisotropy field $H_{\mathrm{K}}$ [33]. All $H_{\mathrm{K}}$ values for films of type A deposited at temperatures in the range $100{ }^{\circ} \mathrm{C}-300^{\circ} \mathrm{C}$ were found to cluster around an average of $14.9 \pm 0.3 \mathrm{kOe}$. This single value of anisotropy field indicates that any structural change in the sample, e.g., due to interfacial intermixing, is too small to significantly affect the effective anisotropy.

To investigate the contributions of the top and bottom Co interfaces to the anisotropy, $H_{\mathrm{K}}$ was also measured for films of types $\mathrm{B}$ and $\mathrm{C}$ in which the deposition temperature of only the top Pt layer was varied and the deposition conditions of the lower interface were kept constant. Figure 6 shows that, for films of type B grown in the usual order (hot to cold), the anisotropy field decreases as $T_{\text {dep }}$ decreases, while for films of type C, grown in reverse (cold to hot), there is very little change in the anisotropy field. Two conclusions may be drawn from this. The first is that annealing the films at $300^{\circ} \mathrm{C}$, as occurs for type $\mathrm{C}$, produces a uniform anisotropy field across the set. (The anisotropy field for type $\mathrm{C}$ is $1-1.5 \mathrm{kOe}$ larger than the anisotropy field for type A, suggesting that annealing improves the perpendicular anisotropy slightly.) The second is that forming the lower Co interface first at a fixed $T_{\text {dep }}$ and subsequently forming the upper interface at successively lower temperatures have the effect of reducing the anisotropy field monotonically from the type-C value. The latter indicates that both upper and lower Co interfaces contribute to the total effective perpendicular anisotropy in all films. The very different $H_{\mathrm{K}}$ for a few of the samples is most likely due to fluctuations in the growth conditions. For 
films of type A, as we have seen, changes in the quality of these interfaces introduced by deposition at different temperatures in the range $100^{\circ} \mathrm{C}-300^{\circ} \mathrm{C}$ have little effect on the perpendicular anisotropy. This is useful because the effect of interface quality on DMI can now be investigated with the perpendicular anisotropy effectively kept constant.

\section{DZYALOSHINSKII-MORIYA INTERACTION}

The interfacial DMI promotes the formation of chiral Néeltype domain walls in films with perpendicular anisotropy. The method of expanding a domain in an in-plane field [4,9] is used to determine the $\mathrm{DMI}$ field in $\mathrm{Pt} / \mathrm{Co} / \mathrm{Pt}$ of type $\mathrm{A}$. Kerr imaging is used in the quasistatic regime: a bubble domain is nucleated via an out-of-plane magnetic field pulse $H_{\mathrm{z}}$, and a background image is taken. A constant in-plane magnetic field $H_{\mathrm{x}}$ is then applied while $H_{\mathrm{z}}$ is pulsed again. The background image is subtracted from the resulting image to show the DW motion [Figs. 7(a)-7(d)] and thus the DW velocity [Figs. 7(e)-7(1)]. The pulse length is of sufficient duration $(>1 \mathrm{~s})$ that the rise time $(<33 \mathrm{~ms})$ is negligible. By repeating for various values of $H_{\mathrm{x}}$ the minimum DW velocity is found, at which point we assume that $H_{\mathrm{x}}=H_{\mathrm{DMI}}$, the effective DMI field. There are reports $[10,34,35]$ that the DMI might not be the only factor behind the shift of the minimum DW velocity away from $H_{\mathrm{x}}=0$, but we assume it is the dominant factor here because the DW velocity vs $H_{\mathrm{x}}$ curves in Figs. 7(e)-7(1) do not exhibit a large asymmetry about the minimum, and the bubble domains [Figs. 7(a)-7(d)] do not show teardrop tendencies. The shift in the minimum DW velocity away from $H_{\mathrm{x}}=0$ is reproduced in a model with $H_{\mathrm{DMI}}$ as the only term that introduces asymmetry. This model is based on a creep law $[36,37]$ with the exponential term dependent on the ratio of domain wall energy densities:

$$
v=v_{0} \exp \left[\zeta\left(\mu_{0} H_{\mathrm{z}}\right)^{-1 / 4}\right],
$$

where

$$
\zeta=\zeta_{0}\left[\epsilon\left(H_{\mathrm{x}}\right) / \epsilon(0)\right]^{1 / 4}
$$

Here $v_{0}$ is the characteristic velocity, $\zeta_{0}$ is a scaling constant, and for a domain wall with mixed Bloch-Néel spin structure the energy density is

$$
\epsilon=\epsilon_{0}-\frac{\pi^{2} \delta \mu_{0}^{2} M_{\mathrm{s}}^{2}}{8 K_{\mathrm{D}}}\left(H_{\mathrm{x}}+H_{\mathrm{DMI}}\right)^{2} .
$$

For a domain wall with pure Néel spin structure the domain wall energy density is

$$
\epsilon=\epsilon_{0}+2 K_{\mathrm{D}} \delta-\pi \delta \mu_{0} M_{\mathrm{S}}\left|H_{\mathrm{x}}+H_{\mathrm{DMI}}\right|,
$$

where the Bloch wall energy density $\epsilon_{0}=2 \pi \sqrt{A K_{0}}$, the exchange stiffness $A=16 \times 10^{-12} \mathrm{~J} / \mathrm{m}$, and $K_{0}$ is the effective anisotropy, used as a fitting parameter; $\delta=\sqrt{A / K_{0}}$ is the domain wall width; $M_{\mathrm{s}}=1.1 \times 10^{6} \mathrm{~A} / \mathrm{m}$ is the saturation magnetization; and $K_{\mathrm{D}}=\left(N_{\mathrm{x}} \mu_{0} M_{\mathrm{s}}^{2}\right) / 2$ is the domain wall anisotropy, where the demagnetizing factor $N_{\mathrm{x}}=\ln (2) t /(\pi \delta)$, with $t$ being the thickness of the ferromagnetic film. Values of saturation magnetization and exchange stiffness were taken from previous work $[4,14]$.

Measuring DW velocity as a function of $H_{\mathrm{z}}$ with no applied $H_{\mathrm{x}}$, we found a linear relationship between $\ln v$ and $H_{\mathrm{z}}^{-1 / 4}$, confirming that the DW motion in the $\mathrm{Pt} / \mathrm{Co} / \mathrm{Pt}$ films was, indeed, in the creep regime. Fitting to $\ln v$ vs $H_{\mathrm{z}}^{-1 / 4}, v_{0}$ and $\zeta_{0}$ were determined, leaving just $H_{\mathrm{DMI}}$ and $K_{0}$ as fitting parameters for velocities measured as a function of $H_{\mathrm{x}}$, shown in Figs. 7(e)-7(h). Values of $K_{0}$ determined from fits to the data in Figs. $7(\mathrm{e})-7(\mathrm{~h})$ ranged from approximately 30 to $60 \mathrm{~kJ} / \mathrm{m}^{3}$, where the upper bound is the effective anisotropy found from the average $H_{\mathrm{K}}$ measured in Sec. IV. The spread in $K_{0}$ may arise as a result of the range of local anisotropies probed by the moving domain wall, as opposed to the single value of anisotropy field measured for the samples as a whole. $H_{\mathrm{DMI}}$, however, is more reliably determined as it controls the asymmetry of the fit.

Data shown in Fig. 7 for a set of films grown at a base pressure of $1.1 \times 10^{-7}$ Torr display the asymmetric expansion of the bubble domains [Figs. 7(a)-7(d)], as well as the shift in minimum of the DW velocity [Figs. 7(e)-7(1)]. A slight change of gradient is apparent when $\log _{10}(v)$ is plotted as a function of $H_{\mathrm{x}}$, indicated by the arrows in Figs. 7(i)-7(1), and in other studies has been attributed to chiral damping [34]. However, the field at which this change of gradient occurs is at roughly the same distance from the minimum as the magnitude of the domain wall shape anisotropy field (310 \pm $90 \mathrm{Oe})$, indicating that at this point the DW spin structure changes from mixed Bloch-Néel to pure Néel. The net DMI fields were found to range between -90 and $276 \mathrm{Oe}$, and so $H_{\mathrm{DMI}}$ alone is not sufficient to bring the domain walls into a pure Néel configuration in any of the films, meaning that at $H_{\mathrm{x}}=0$ the domain wall spin structure is mixed Bloch-Néel. The DW velocities in Figs. 7(e)-7(h) were fitted by Eq. (1) with the DW energy density (mixed Bloch-Néel or pure Néel) chosen according to whether $H_{\mathrm{x}}$ was greater than or less than the DW shape anisotropy field.

Figure 8 shows $H_{\mathrm{DMI}}$ for sets of films grown at a range of base pressures from $1.1 \times 10^{-7}$ to $3.3 \times 10^{-7}$ Torr plotted against the normalized difference in $\sigma$ for the upper and lower Co interfaces. The difference in $\sigma$ is a measure of the difference in quality of the upper and lower Co interfaces. Measuring the net DMI field for several sets of samples permits a correlation to emerge, as the DMI is exquisitely sensitive to the difference in quality of the upper and lower Co interfaces. The difference in $\sigma$ is normalized by the total $\sigma$ to highlight any dependence on the interface quality difference, independent of any changes in total $\sigma$.

Figure 8 shows that the net DMI in epitaxial $\mathrm{Pt} / \mathrm{Co} / \mathrm{Pt}$ increases from zero to positive values when the quality of the top interface decreases relative to the bottom and decreases from zero to negative values when the quality of the bottom interface decreases relative to the top. A linear fit to the data yields a Pearson's $r$ correlation coefficient of 0.52 . This indicates a moderate correlation [38] between net DMI and relative interface quality, in spite of the scatter that could arise mainly from fluctuations in the base pressure and deposition temperature and the uncertainty in $\sigma$ for individual interfaces. While the scatter means we do not have perfect control over the properties of a film using this method, there is sufficient correlation that the majority of data points are fairly close to the fitted line. Sensitive interface effects are particularly difficult to control when, as here, there is more than one interface to consider. Given this, the correlation goes a long way towards 
(a)

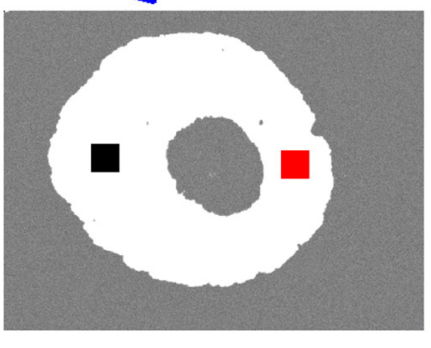

(b)

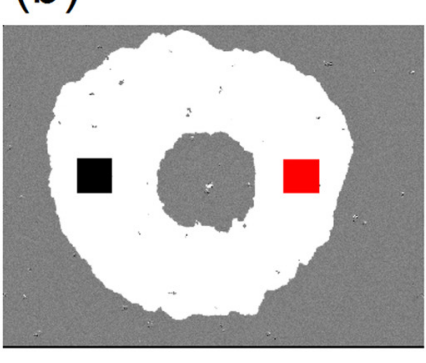

(c)

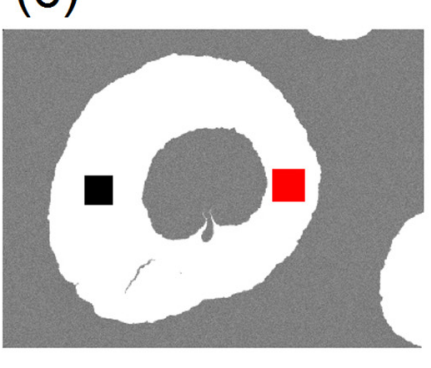

(d)

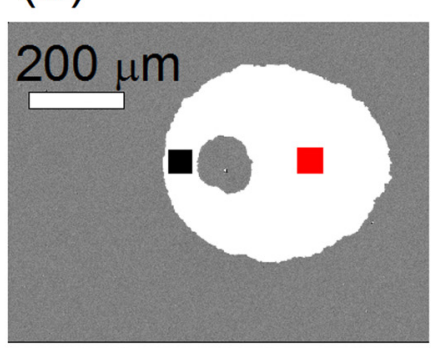

(e)

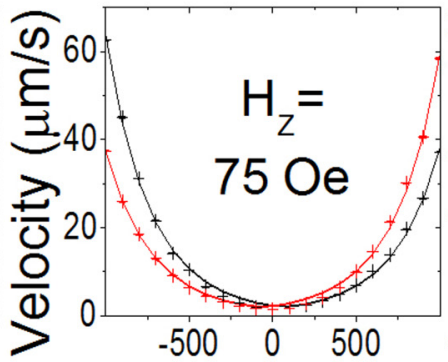

(f)

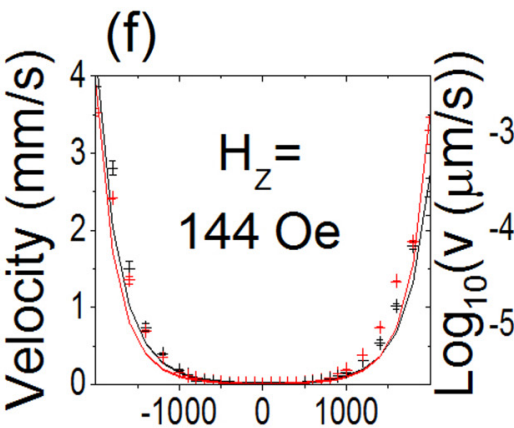

(i)

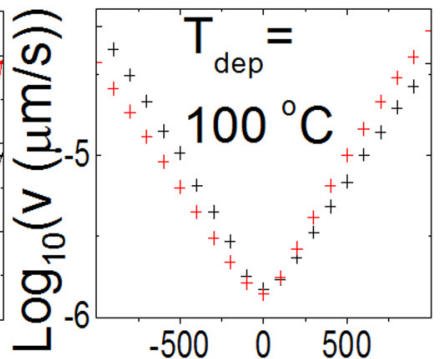

(j)

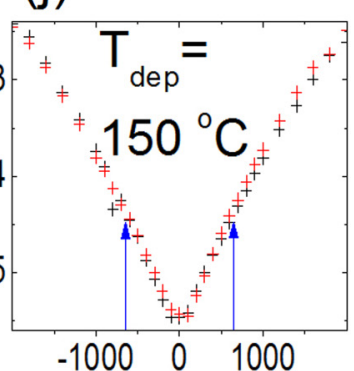

(g) $(\mathrm{k})$

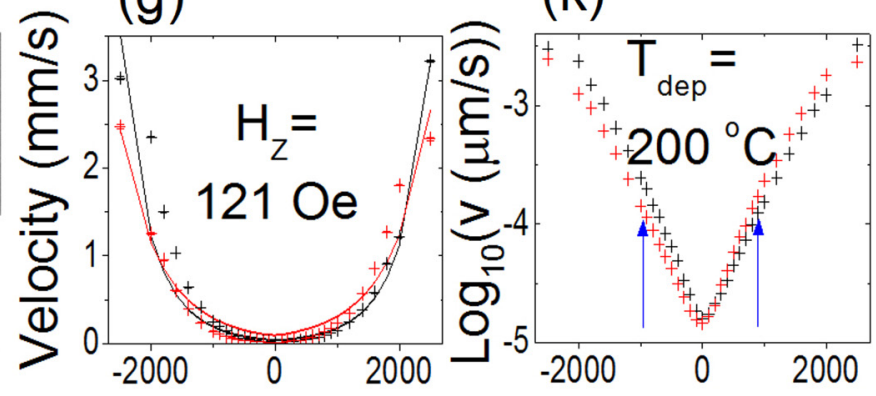

(h)

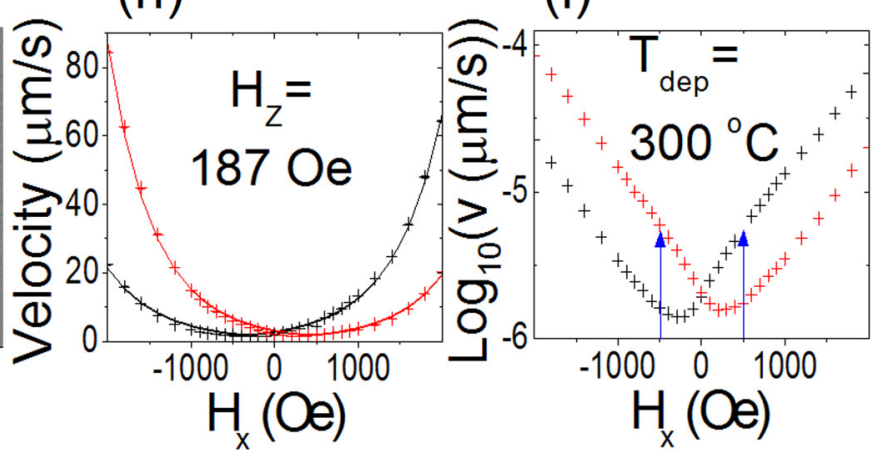

FIG. 7. (a) to (d) Differential-mode Kerr microscope images of bubble domains used to determine domain wall displacements in a field of $H_{\mathrm{x}}=-1000$ Oe for a set of $\mathrm{Pt} / \mathrm{Co} / \mathrm{Pt}$ films grown at low base pressure $\left(1.1 \times 10^{-7}\right.$ Torr $)$ and deposition temperatures indicated for each row. (e) to (h) DW velocities fitted by Eq. (1) and (i) to (1) the logarithm of DW velocity as a function of $H_{\mathrm{x}}$. $H_{\mathrm{z}}$ is the out-of-plane pulsed field used to expand the domain. Black and red correspond to the left- and right-moving walls of the domain, respectively, and the blue arrows indicate the domain wall anisotropy field.

understanding and separating the relative effects of the two interfaces.

The conclusion that may be drawn from Fig. 8 is that the net DMI field in epitaxial $\mathrm{Pt} / \mathrm{Co} / \mathrm{Pt}$ can change by up to $400 \pm$ 100 Oe depending on the deposition conditions. For less ordered, polycrystalline $\mathrm{Pt} / \mathrm{Co} / \mathrm{Pt}$, therefore, it is no surprise that larger DMI fields may be obtained; for example, Franken et al. [39] obtained $H_{\text {DMI }}=370 \pm 10$ Oe in a polycrystalline stack with a thinner Co layer (4 $\AA$ ), and Hrabec et al. [4] obtained $H_{\mathrm{DMI}} \sim-1000$ Oe for films with a similar Co thickness but that were polycrystalline and had a different Pt thickness. Naively mapping $H_{\mathrm{DMI}} \sim-1000$ Oe onto Fig. 8 


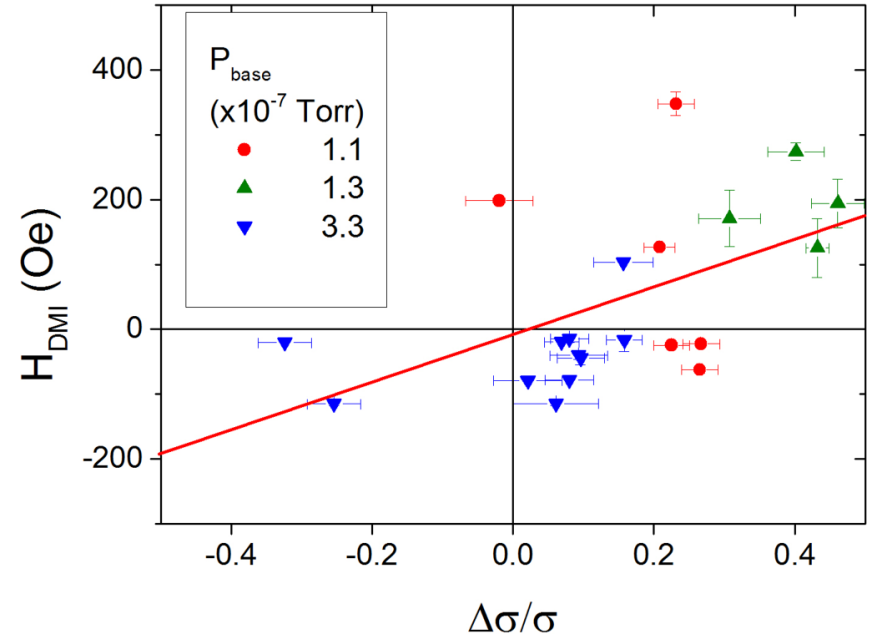

FIG. 8. Net $H_{\mathrm{DMI}}$ as a function of $\Delta \sigma / \sigma$, the difference between the top Co interface $\sigma$ and the bottom Co interface $\sigma$ for epitaxial $\mathrm{Pt} / \mathrm{Co} / \mathrm{Pt}$ deposited at temperatures in the range $100^{\circ} \mathrm{C}-300^{\circ} \mathrm{C}$, normalized by the total $\sigma$. A positive (negative) $\Delta \sigma / \sigma$ corresponds to the lower (upper) Co interface being a higher quality than the upper (lower) one. The errors associated with $\Delta \sigma / \sigma$ relate to the suitability of the $\sigma$ values to the fitted x-ray reflectivity models and so act as a lower bound of uncertainty due to the deviations of the fits. The solid line is a linear fit to the data.

yields a difference in $\Delta \sigma / \sigma$ of -2.5 , which would mean that the difference in interface quality is greater than the total interface quality and suggests that there is another factor at work here, possibly related to the polycrystallinity.

For samples of type A where growth was initiated at base pressures at the lower end of the range studied here, $H_{\mathrm{DMI}}$ increased monotonically as a function of $T_{\mathrm{dep}}$, as shown in Fig. 9. As the base pressure increases, the temperature has less influence on the interface quality, and thus, the range of $H_{\mathrm{DMI}}$ values decreases and becomes less obviously linear in temperature. Using the prior finding that the lower Co interface contributes a positive $H_{\mathrm{DMI}}$ [4] and the conclusion that a higher-quality (smaller $\sigma$ ) interface contributes a larger $H_{\text {DMI }}$, the increase of $H_{\text {DMI }}$ with $T_{\text {dep }}$ may be interpreted as follows. As $T_{\text {dep }}$ increases, the quality of both interfaces, but particularly the upper one, decreases until annealing dominates and their quality improves. This difference in interface quality introduces structural inversion asymmetry, necessary for a net DMI field to occur, increasing as the contribution from the top interface is reduced, as seen in Fig. 9. As the Co interfaces become of a similar quality, the DMI contributions from the

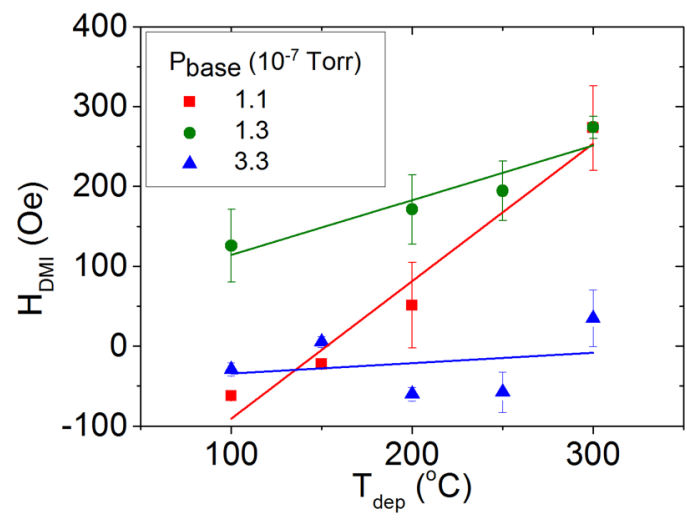

FIG. 9. Net $H_{\mathrm{DMI}}$ as a function of $T_{\mathrm{dep}}$ for epitaxial $\mathrm{Pt} / \mathrm{Co} / \mathrm{Pt}$ where growth was initiated at various base pressures. The solid lines are linear fits to the data. The error bars are the larger of either statistical standard error or error in velocity vs $H_{\mathrm{x}}$ fits.

top and bottom cancel, causing no net DMI. If the bottom interface is of a lower quality than the top one, the dominant contribution switches, and the effective DMI field becomes negative. This shows that if the base pressure is at the lower end of the range studied here, substrate temperature may be used to linearly adjust the DMI.

\section{CONCLUSION}

In conclusion, changing the temperature during deposition of the top $\mathrm{Co} / \mathrm{Pt}$ layers in an epitaxially sputtered $\mathrm{Pt} / \mathrm{Co} / \mathrm{Pt}$ system significantly affects the Co interface quality. The difference between the quality of the top interface and that of the lower interface introduces structural inversion asymmetry which results in a net DMI field. This difference in interface quality is altered with deposition temperature, and it is thus shown that, for the lowest base pressures in the range studied, the substrate temperature may be used to fine-tune the DMI in epitaxial samples.

The data associated with this paper are openly available from the University of Leeds Data Repository (http://doi.org/10.5518/52).

\section{ACKNOWLEDGMENTS}

This work was supported by the U.K. EPSRC (Grants No. EP/I011668/1, No. EP/K003127/1, and No. EP/M000923/1).
[1] S. Pizzini, J. Vogel, S. Rohart, L. D. Buda-Prejbeanu, E. Jué, O. Boulle, I. M. Miron, C. K. Safeer, S. Auffret, G. Gaudin, and A. Thiaville, Phys. Rev. Lett. 113, 047203 (2014).

[2] M. Belmeguenai, J.-P. Adam, Y. Roussigné, S. Eimer, T. Devolder, J.-V. Kim, S. M. Cherif, A. Stashkevich, and A. Thiaville, Phys. Rev. B 91, 180405(R) (2015).
[3] M. J. Benitez, A. Hrabec, A. P. Mihai, T. A. Moore, G. Burnell, D. McGrouther, C. H. Marrows, and S. McVitie, Nat. Commun. 6, 8957 (2015).

[4] A. Hrabec, N. A. Porter, A. Wells, M. J. Benitez, G. Burnell, S. McVitie, D. McGrouther, T. A. Moore, and C. H. Marrows, Phys. Rev. B 90, 020402(R) (2014). 
[5] H. Yang, O. Boulle, V. Cros, A. Fert, and M. Chshiev, arXiv:1603.01847.

[6] T. Koyama, D. Chiba, K. Ueda, K. Kondou, H. Tanigawa, S. Fukami, T. Suzuki, N. Ohshima, N. Ishiwata, Y. Nakatani, K. Kobayashi, and T. Ono, Nat. Mater. 10, 194 (2011).

[7] K.-S. Ryu, L. Thomas, S.-H. Yang, and S. Parkin, Nat. Nanotechnol. 8, 527 (2013).

[8] G. Chen, T. Ma, A. T. N'Diaye, H. Kwon, C. Won, Y. Wu, and A. K. Schmid, Nat. Commun. 4, 2671 (2013).

[9] S.-G. Je, D.-H. Kim, S.-C. Yoo, B.-C. Min, K.-J. Lee, and S.-B. Choe, Phys. Rev. B 88, 214401 (2013).

[10] R. Lavrijsen, D. M. F. Hartmann, A. van den Brink, Y. Yin, B. Barcones, R. A. Duine, M. A. Verheijen, H. J. M. Swagten, and B. Koopmans, Phys. Rev. B 91, 104414 (2015).

[11] M. Vaňatka, J.-C. Rojas-Sánchez, J. Vogel, M. Bonfim, M. Belmeguenai, Y. Roussigné, A. Stashkevich, A. Thiaville, and S. Pizzini, J. Phys. Condens. Matter 27, 326002 (2015).

[12] F. C. Ummelen, D.-S. Han, J.-S. Kim, H. J. M. Swagten, and B. Koopmans, IEEE Trans. Magn. 51, 1 (2015).

[13] S. Emori, U. Bauer, S.-M. Ahn, E. Martinez, and G. S. D. Beach, Nat. Mater. 12, 611 (2013).

[14] A. Thiaville, S. Rohart, E. Jué, V. Cros, and A. Fert, Europhys. Lett. 100, 57002 (2012).

[15] J. Sampaio, V. Cros, S. Rohart, A. Thiaville, and A. Fert, Nat. Nanotechnol. 8, 839 (2013).

[16] W. Jiang, P. Upadhyaya, W. Zhang, G. Yu, M. B. Jungfleisch, F. Y. Fradin, J. E. Pearson, Y. Tserkovnyak, K. L. Wang, O. Heinonen, S. G. E. te Velthuis, and A. Hoffmann, Science 349, 283 (2015).

[17] C. Moreau-Luchaire, C. Moutafis, N. Reyren, J. Sampaio, C. Vaz, N. Van Horne, K. Bouzehouane, K. Garcia, C. Deranlot, P. Warnicke, P. Wohlhüter, J.-M. George, M. Weigand, J. Raabe, V. Cros, and A. Fert, Nat. Nanotechnol. 11, 444 (2016).

[18] S. Woo, K. Litzius, B. Krüger, M.-Y. Im, L. Caretta, K. Richter, M. Mann, A. Krone, R. Reeve, M. Weigand, P. Agrawal, I. Lemesh, M.-A. Mawass, P. Fischer, M. Kläui, and G. Beach, Nat. Mater. 15, 501 (2016).

[19] O. Boulle, J. Vogel, H. Yang, S. Pizzini, D. de Souza Chaves, A. Locatelli, T. Mentes, A. Sala, L. Buda-Prejbeanu, O. Klein, M. Belmeguenai, Y. Roussigné, A. Stashkevich, S. Chérif, L. Aballe, M. Foerster, M. Chshiev, S. Auffret, I. Miron, and G. Gaudin, Nat. Nanotechnol. 11, 449 (2016).

[20] W. Kang, Y. Huang, C. Zheng, W. Lv, N. Lei, Y. Zhang, X. Zhang, Y. Zhou, and W. Zhao, Sci. Rep. 6, 23164 (2016).
[21] S. S. P. Parkin, M. Hayashi, and L. Thomas, Science 320, 190 (2008).

[22] W. Kang, Y. Huang, C. Zheng, W. Lv, N. Lei, Y. Zhang, X. Zhang, Y. Zhou, and W. Zhao, Sci. Rep. 6, 9400 (2015).

[23] D. A. Allwood, G. Xiong, C. C. Faulkner, D. Atkinson, D. Petit, and R. P. Cowburn, Science 309, 1688 (2005).

[24] A. R. Fert, Mater. Sci. Forum 59-60, 439 (1990).

[25] K.-W. Moon, D.-H. Kim, S.-C. Yoo, S.-G. Je, B. S. Chun, W. Kim, B.-C. Min, C. Hwang, and S.-B. Choe, Sci. Rep. 5, 9166 (2015).

[26] D. Petit, P. R. Seem, M. Tillette, R. Mansell, and R. P. Cowburn, Appl. Phys. Lett. 106, 022402 (2015).

[27] See Supplemental Material at http://link.aps.org/supplemental/ 10.1103/PhysRevB.95.054428 for further information about the sputtering environment and the relationship between the Dzyaloshinskii-Moriya interaction field and the base pressure, respectively working pressure.

[28] A. P. Mihai, A. L. Whiteside, E. J. Canwell, C. H. Marrows, M. J. Benitez, D. McGrouther, S. McVitie, S. McFadzean, and T. A. Moore, Appl. Phys. Lett. 103, 262401 (2013).

[29] S. K. Sinha, E. B. Sirota, S. Garoff, and H. B. Stanley, Phys. Rev. B 38, 2297 (1988).

[30] D. E. Savage, J. Kleiner, N. Schimke, Y.-H. Phang, T. Jankowski, J. Jacobs, R. Kariotis, and M. G. Lagally, J. Appl. Phys. 69, 1411 (1991).

[31] M. Wormington, C. Panaccione, K. M. Matney, and D. K. Bowen, Philos. Trans. R. Soc. London, Ser. A 357, 2827 (1999).

[32] F. Bolzoni, F. Leccabue, R. Panizzieri, and L. Pareti, IEEE Trans. Magn. 20, 1625 (1984).

[33] K.-W. Moon, J.-C. Lee, S.-B. Choe, and K.-H. Shin, Rev. Sci. Instrum. 80, 113904 (2009).

[34] E. Jué, C. K. Safeer, M. Drouard, A. Lopez, P. Balint, L. BudaPrejbeanu, O. Boulle, S. Auffret, A. Schuhl, A. Manchon, I. M. Miron, and G. Gaudin, Nat. Mater. 15, 272 (2016).

[35] D. Lau, V. Sundar, J.-G. Zhu, and V. Sokalski, Phys. Rev. B 94, 060401(R) (2016).

[36] F. Cayssol, D. Ravelosona, C. Chappert, J. Ferré, and J. P. Jamet, Phys. Rev. Lett. 92, 107202 (2004).

[37] P. J. Metaxas, J. P. Jamet, A. Mougin, M. Cormier, J. Ferré, V. Baltz, B. Rodmacq, B. Dieny, and R. L. Stamps, Phys. Rev. Lett. 99, 217208 (2007).

[38] J. D. Evans, Straightforward Statistics for the Behavioral Sciences (Brooks/Cole, Pacific Grove, CA, 1996).

[39] J. H. Franken, M. Herps, H. J. M. Swagten, and B. Koopmans, Sci. Rep. 4, 5248 (2014). 